\title{
Global optimisation methods for poroelastic material characterisation using a clamped sample in a Kundt tube setup
}

\author{
Johan Vanhuyse ${ }^{1, *}$, Elke Deckers, Stijn Jonckheere, Bert Pluymers, Wim Desmet \\ KU Leuven - Dept. of Mechanical Engineering, Celestijnenlaan 300B - box 2420, 3001 Leuven, Belgium
}

\begin{abstract}
The Biot theory is commonly used for the simulation of the vibro-acoustic behaviour of poroelastic materials. However, it relies on a number of material parameters which can be hard to characterise. These parameters are nowadays often determined using dedicated measurement setups for each parameter, yielding a time-consuming and costly characterisation. This paper presents a characterisation method which is able to identify all material parameters using only an impedance tube. The method relies on the assumption that the sample is clamped within the tube, that the shear wave is excited and that the acoustic field within the tube is no longer one-dimensional. The effect of the material on the sound field is quantified using reflection coefficients or relative pressures. This paper numerically shows the potential of the developed method. It therefore performs a sensitivity analysis of the quantification parameters and a parameter estimation using global optimisation methods. A new 3-step procedure is developed and validated to yield robust parameter estimations. The effect of numerically simulated noise on the robustness on the parameter estimation is also discussed. It is shown that even in the presence of this noise the developed procedure leads to a robust parameter estimation.
\end{abstract}

Keywords: parameter estimation, poroelastic Biot theory, optimisation, Kundt tube, Wave Based Method

\section{Introduction}

Due to increasingly restrictive legal regulations and increasing customer demands, the vibro-acoustic behaviour of products becomes a topic of high importance in for example the automotive sector and industrial machinery environments. The quest for lighter and hybrid cars to reduce the fuel consumption and the ecological footprint of the vehicles in general, inherently comes with deteriorated noise and vibration properties [1]. Therefore, these industries typically use poroelastic materials to keep noise limited to acceptable levels. Besides, the same quality has to be delivered in ever shorter time-to-market cycles. Therefore, the use of virtual prototyping tools has become an essential part of the design process.

Both aspects enlarge the need for a reliable simulation of the effect of poroelastic materials on the sound field. For vibro-acoustic simulations, the Biot theory [2], as adapted by Johnson et al. [3], Champoux and Allard [4] (JCA), is commonly used [5] and describes the dynamic behaviour of the poroelastic material by two coupled partial differential equations. The model applies a homogenised solid and a compressible fluid continuum description on a macroscopic level. It has been shown that this homogenisation is justified if the pore dimensions are much smaller than the wavelengths of the different waves propagating through the material. The Biot theory predicts the existence of three waves which propagate in both phases simultaneously: two longitudinal waves and one shear wave.

Different numerical procedures such as Finite Element formulations e.g. [6, 7, 8], the Transfer Matrix Method [9], Trefftz approaches [10, 11, 12] and hybrid approaches [13] have been developed to predict the outcome of vibro-acoustic problem settings including poroelastic materials. Each method and formulation has its advantages

\footnotetext{
${ }^{*}$ E-mail address: johan.vanhuyse@kuleuven.be

${ }^{1}$ Present address: Siemens Industry Software NV, Interleuvenlaan 68, 3001 Leuven, Belgium
} 
and drawbacks, but the accuracy of the simulations using each of them logically relies on the accuracy of the material properties.

To describe the behaviour of the poroelastic material, the JCA model of Biot's theory uses a number of material dependent parameters, which can be related to properties of the frame material, properties of the fluid filling the voids, and the geometry of the pores, respectively. The standard properties of air are assumed to be known in this paper. The geometry of the pores determines the porosity $\phi$, the tortuosity $\alpha_{\infty}$, the flow resistance $\sigma$, the viscous characteristic length $\Lambda$ and the thermal characteristic length $\Lambda^{\prime}$. Furthermore, the bulk density of the skeleton $\rho_{1}$, the complex Young's modulus of the frame in vacuum $E$ and the complex shear modulus of the frame $N$ depend on the solid constituent.

Currently, there are different approaches to obtain the above mentioned parameters. For all parameters, dedicated tests and associated measurement setups, advantages and disadvantages, have been discussed in literature, see e.g. $[5,14,15,16,17]$ and references therein. Parameters such as the porosity and airflow resistivity can be easily measured using standard techniques, while the others show to be more difficult to determine with sufficient accuracy. Besides, due to the use of different test setups, the characterisation of poroelastic materials is costly and time consuming. Other authors propose to determine multiple parameters at once, using optimisation strategies. The main example is the procedure described in [18], which applies an equivalent fluid model and uses Kundt tube measurements to determine the characteristic lengths and the tortuosity. This method has also been commercially implemented in FOAM-X, which in addition allows to extract $\phi$ and $\sigma$ [19]. The use of transmission tube measurements to retrieve all parameters at once has been proposed in [20], however, large differences with respect to reference values are sometimes observed and it is indicated that the constrained edge of the sample may have an influence.

As has been shown in [21], round-robin tests to validate the reproducibility on measurements of the acoustic impedance and absorption of porous foams using a Kundt tube show a large spread on the results. It is indicated that specification of sample preparation and sample support should be included in the measurement procedure. This illustrates the sensitivity of measurement results to the test setup.

When considering impedance tube measurements, as has been shown in various studies, e.g. see [22, 23, 24], the mounting conditions can have an important impact on the results. When the sample is clamped into the tube, also the shear wave is excited and the sample has a stiffer behaviour. To account for these effects, analytical expressions are insufficient to accurately predict the dynamic behaviour in certain frequency ranges and numerical prediction techniques should be applied. When impedance tube measurements are applied for inverse material characterisation, analytical expressions are used, applying equivalent fluid models [18] or Biot models [20]. In the former case often needles are inserted in the samples to rigidify the porous material. This way, the effect of solid phase on the material sample is minimised and the behaviour of the poroelastic material better approximates the equivalent fluid behaviour. In the latter case it is presumed that sliding edge boundary conditions [25] are valid and that the sample is loosely fitting in the tube.

Recently an efficient numerical method has been developed which allows modelling an impedance test setup in which the sample is clamped into the tube [26]. The main goal of this paper is to exploit the fact that the sound field within the measurement tube is not one-dimensional when the sample is clamped into the tube. It will be shown, given the assumption that fixed edge boundary conditions accurately represent the physical behaviour of the sample, it is possible to retrieve all poroelastic material parameters, performing one single measurement campaign and using a widely available and low cost impedance tube. Besides, it is validated whether the number and location of the microphones can be optimised to obtain a better convergence.

This paper is organised as follows. Section 2 describes the developed procedure and the numerical model used to support this procedure. Section 3 explains the assets of using a 2D model and describes the parameters used to quantify the sound field in the Kundt tube. This quantification is necessary to perform a parameter estimation using optimisation methods. Section 4 assesses the sensitivity of the different quantification parameters to the material parameters. This section also discusses the frequency dependency and the spatial dependency of the sensitivity. Section 5 assesses the use of global optimisation methods for parameter estimation. These optimisation methods use multiple starting points to obtain a better performance than local optimisation methods. A more robust parameter estimation procedure is presented. Section 6 describes the effect of noise on the optimisation performance. The paper concludes with a number of final remarks. 


\section{Problem description}

This paper investigates whether a Kundt tube measurement setup can be used (with minor adaptations) in combination with efficient numerical simulations using the Wave Based Method (WBM) to estimate all parameters of a poroelastic material, associated with both the fluid and the solid phase. To this extent, this section first briefly explains the Biot theory of poroelasticity. Thereafter, a short description of the measurement setup and the WBM model are given.

\subsection{Poroelastic materials}

The Biot theory $[2,5]$ is most commonly applied to model the mutually coupled dynamic behaviour of the skeleton and the interpenetrating fluid inside a poroelastic material. It applies a homogenisation of the material into a separate solid and fluid continuum description which holds as long as the wavelengths of the propagating waves (two longitudinal and one shear wave) are much larger than the pore size. The Biot equations, which follow from the momentum equations and the constitutive relations, can be written as two coupled, vectorial, partial differential equations:

$$
\begin{aligned}
N \nabla^{2} \mathbf{u}^{\mathrm{s}}(\mathbf{r})+\nabla\left[(\tilde{A}+N) e^{s}(\mathbf{r})+\tilde{Q} e^{f}(\mathbf{r})\right] & =-\omega^{2}\left(\tilde{\rho}_{11} \mathbf{u}^{\mathrm{s}}(\mathbf{r})+\tilde{\rho}_{12} \mathbf{u}^{\mathbf{f}}(\mathbf{r})\right), \\
\nabla\left[\tilde{Q} e^{s}(\mathbf{r})+\tilde{R} e^{f}(\mathbf{r})\right] & =-\omega^{2}\left(\tilde{\rho}_{12} \mathbf{u}^{\mathrm{s}}(\mathbf{r})+\tilde{\rho}_{22} \mathbf{u}^{\mathbf{f}}(\mathbf{r})\right),
\end{aligned}
$$

with $\mathbf{u}^{\mathrm{s}}(\mathbf{r})$ and $\mathbf{u}^{\mathbf{f}}(\mathbf{r})$ the unknown vectorial deformation fields of the solid and fluid phase respectively and $e^{\bullet}(\mathbf{r})=$ $\nabla \cdot \mathbf{u}^{\bullet}(\mathbf{r})$ the respective volumetrical strains. The Biot equations are complemented by a set of three conditions for each point on the boundary. These can be either sliding edge conditions (zero normal deformations in the solid and fluid phase and zero shear stress in the solid phase), fixed edge conditions (zero normal deformations in the solid and fluid phase and zero tangential deformations in the solid phase) or free edge conditions (zero normal stress in the solid and fluid phase and zero shear stress in the solid phase).

The material parameters of the solid skeleton are given by the shear modulus $N$ and the first Lamé coefficient in air $\tilde{A}$, which can be derived from the frame Young's modulus $E$. The behaviour of the interpenetrating fluid is modelled by the effective bulk modulus, which is proportional to $\tilde{R}$. Both phases are mutually coupled by a factor $\tilde{Q}$. The inertial and viscous damping effects are comprised in the density terms $\tilde{\rho}_{11}$ (derived from the skeleton bulk density $\rho_{1}$ ), a coupling term $\tilde{\rho}_{12}$ and $\tilde{\rho}_{22}$ (derived from the fluid bulk density $\rho_{0}$ ).

The most often used theory to determine these parameters $\tilde{R}=(1-\phi) \tilde{K}_{f}, \tilde{Q}=\phi \tilde{K}_{f}, \tilde{\rho}_{11}, \tilde{\rho}_{12}$ and $\tilde{\rho}_{22}$ is the semi-phenomenological model of Johnson et al. [3], Champoux and Allard [4], which assumes that the air saturating the porous material has a low compressibility compared to the skeleton compressibility. In this modelling framework, the determining parameters can be calculated from a frequency independent set of parameters describing the bulk properties of the pores. These are the porosity $\phi$, describing the volume fraction of fluid phase to the overall volume, the bulk density of the frame $\rho_{1}$, the tortuosity $\alpha_{\infty}$, taking into account the true acoustic propagation length due to the curvature of the pores, the flow resistance $\sigma$ and the viscous and thermal characteristic length, $\Lambda$ and $\Lambda^{\prime}$ respectively, describing the scale on which either viscous or thermal dissipation mechanisms act. A poroelastic material can thus be modelled with the Biot equations using the JCA assumptions, apart from the standard air properties which are assumed to be known, 3 solid phase parameters and 5 parameters describing the homogenised pore geometry and distribution.

Further information on the JCA model of Biot's theory can be found in the reference textbook by Allard and Atalla [5].

\subsection{Measurement setup}

The Kundt tube (Fig. 1) is a conventional test setup that allows for easy characterisation of absorptive materials. In this cylindrical tube, an acoustic pressure field is generated by a speaker, situated on one end. The sound field is influenced by a material sample placed on the other end. Through measurement of the sound pressure, a general assessment of the absorptive characteristics of this material sample can be made. When the acoustic wavelength is larger than 1.73 times the tube diameter [30], higher order modes will fit the cross-section of the tube, causing unwanted non-plane wave modes propagating in the tube. Below this frequency, the acoustic field inside the tube can be assumed to be 1D. This assumption roughly holds up to $4.9 \mathrm{kHz}$. Below this frequency, the pressure field inside the tube can be decomposed into an incoming and a reflected wave. The ISO-standard [30] also sets a lower 
frequency of validity. If the wavelengths are too long, no clear pressure difference can be measured at the microphone location. This frequency is driven by the distance between the microphones and is be around $340 \mathrm{~Hz}$ for the given setup. The ratio of the amplitudes of both defines the reflection coefficient $R$, which can be determined from the measured acoustic pressures at two microphone locations:

$$
R=\frac{e^{j k x_{1}}-e^{j k x_{2}} p_{1} / p_{2}}{e^{-j k x_{2}} p_{1} / p_{2}-e^{-j k x_{1}}},
$$

with $x_{1}$ and $x_{2}$ the distance of the first and second microphone to the sample and $p_{1}$ and $p_{2}$ the pressures measured by these microphones. From this reflection coefficient, the absorption value $\alpha$ of the sample can be straightforwardly calculated:

$$
\alpha=1-|R|^{2} .
$$

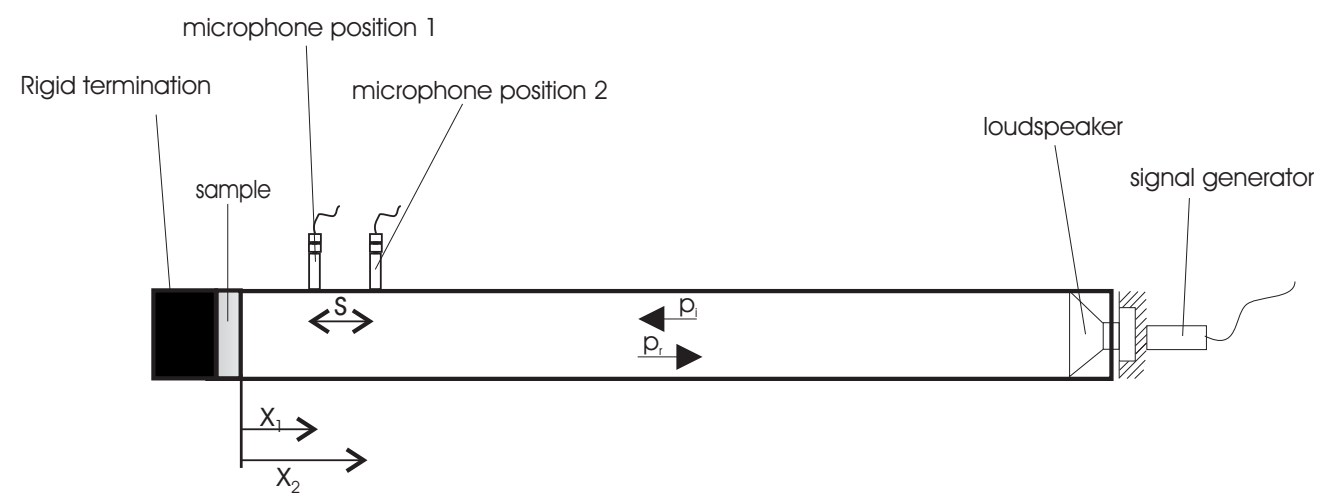

Figure 1: Sketch of a typical Kundt tube measurement setup.

However, a purely 1D pressure field is almost impossible to replicate since the effect of the boundary conditions on the sample cannot be neglected. The $1 \mathrm{D}$ behaviour only holds for sliding edge boundary conditions. In all other boundary condition sets, the shear wave is excited as well. In practice, there is always a degree of clamping of the sample. As a result, the real boundary conditions are somewhere in between sliding edge and fixed edge boundary conditions. In this case, the underlying differential equations have to be used rather than the derived reflection coefficient $R$. For the poroelastic material sample, the Biot equations (1)-(2) are used. The acoustic field inside the cylindrical cavity is described by the well-known acoustic Helmholtz equation:

$$
\nabla^{2} p(\mathbf{r})+k^{2} p(\mathbf{r})=0
$$

in which $p$ is the acoustic pressure and $k$ is the acoustic wave number. This equation is complemented with one condition for each point on the boundary, prescribing pressure (Dirichlet), normal velocity (Neumann) or normal impedance (Robin).

In this paper, a Kundt tube with a diameter of $40 \mathrm{~mm}$ and a length of $100 \mathrm{~mm}$ will be used. In this, the effect of a rigidly backed sample with a thickness of $5 \mathrm{~mm}$ is assessed using two microphones which are located at a distance of $x_{1}=89 \mathrm{~mm}$ and $x_{2}=139 \mathrm{~mm}$ from the sample. Further information on this setup can be found in [22].

\subsection{Numerical model}

Since the Kundt tube has a simple cylindrical geometry, the recently developed axisymmetric Wave Based Method (WBM) is perfectly suited for this problem because of its high efficiency [26]. The WBM [27, 28] is a numerical modelling technique for solving steady-state dynamic problems described by a (set of) Helmholtz equation(s). Contrarily to conventional element-based approaches, which finely discretise the problem domain and apply polynomial approximation functions, the WBM subdivides the problem into large convex subdomains. Within these domains, the solution 
is approximated by so-called wave functions, which are exact solutions to the governing differential equation(s). Although the wave functions satisfy the governing differential equations a priori, they can still violate the boundary and interface condition(s). Using a weighted residual Galerkin approach, these errors are minimised, leading to a system of equations in the unknown wave function contributions. After solution of the system of equations, these can be post-processed into any field variable of interest.

In the model, the cylindrical acoustic cavity of the Kundt tube is the first subdomain. Within this domain, wave functions which satisfy the acoustic Helmholtz equation are used. The boundary conditions are all Neumann conditions: zero normal velocity for the rigid boundary and unit normal velocity as excitation. The second subdomain covers the poroelastic sample. According to the procedure described in [10], the Biot equations are first decoupled into three Helmholtz equations. The dynamic variables of these three decoupled Helmholtz equations are then again approximated by three sets of wave functions. The boundary conditions in this case are either sliding edge and fixed edge conditions. On the acoustic-poroelastic interface, continuity is enforced between the normal stress and the normal deformations on both sides of the interface. The tangential stress in the solid phase is zero here. The back of the poroelastic sample is considered clamped (i.e. rigid backing).

All this leads to the coupled acoustic-poroelastic model shown in Fig. 2. Further information and more theoretical background on the model can be found in [26].

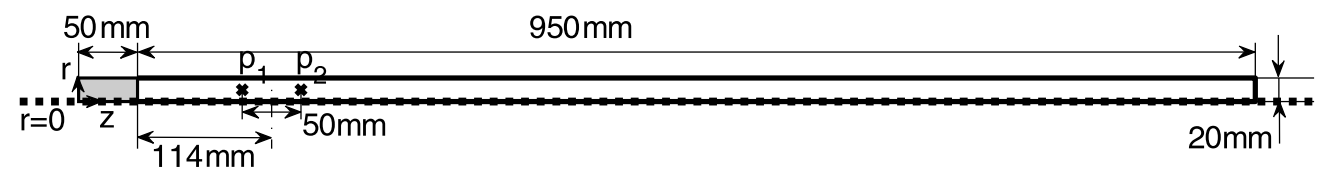

Figure 2: Axisymmetric WBM model of the Kundt tube with a $5 \mathrm{~mm}$ sample.

\section{Quantification of the effect of the material}

In order to perform an estimation of the material parameters, the effect of the poroelastic material on the sound field needs to be evaluated using a quantification parameter. This section first discusses the radial variation of the sound field. This variation needs to be taken into account when the quantification parameters are defined. Secondly, this section discusses different possibilities for the quantification parameters.

Most parameter characterisation methods start from the assumption of 1D behaviour of the sample and the sound field in the Kundt tube. This assumption is not always valid, as has been discussed in section 1. Since in practice a poroelastic sample is at least partly clamped in the tube, or important air gaps between the sample and the Kundt tube exist [24, 29], the sound field will show a radial variation. Indeed, when the poroelastic material is (partly) clamped, the center of it will be able to move, while the edges must remain fixed. This leads to a variation of the sound field in the radial direction. Air gaps between the sample and the Kundt tube give rise to acoustic leakage and thus need to be absolutely avoided in measurements. Therefore, the diameter of the sample is always taken slightly larger than the Kundt tube diameter, which leads to a (at least partially) clampled sample.

Figure 3(a) illustrates the difference in absorption coefficient prediction between sliding edge and fixed edge boundary conditions as calculated for a $5 \mathrm{~cm}$ eurocell sample. The backside of the sample is always considered fixed. Appendix A gives the properties of eurocell. This figure shows that the absorption coefficient $\alpha$ has a different behaviour when fixed edge boundary conditions are used instead of sliding edge boundary conditions. This difference is clearly visible at rather low frequencies $(200 \mathrm{~Hz}-2000 \mathrm{~Hz})$, while it disappears at higher frequencies. Indeed, due to the smaller wavelengths at higher frequencies, the effect of the boundary conditions on the sound field is smaller.

Figure 3(b) illustrates the radial variation of the sound field in a different way. This figure shows the logarithm of $\hat{p}(z)$ as a function of the axial coordinate $z$ for eurocell and fixed edge boundary conditions. A quantification $\hat{p}(z)$ of the spatial deviation relative to a 1D field can be obtained by taking the rms value of the deviation of the sound field from the mean value at each radial location. This value $\hat{p}(z)$ can be calculated as follows, with $N=100$ :

$$
\hat{p}(z)=\sqrt{\sum_{i=1}^{N}\left(\frac{p\left(r_{i}, z\right)-\bar{p}(z)}{\bar{p}(z)}\right)^{2}} \text {, with } \bar{p}(z)=\frac{1}{N} \sum_{i=1}^{N} p\left(r_{i}, z\right) .
$$


In these equations, $p\left(r_{i}, z\right)$ is the spatial pressure field in the Kundt tube, and $\bar{p}(z)$ is the average of the pressures in the radial direction for a given axial location $z$. This figure shows that the radial variation of the spatial sound field is mainly limited to the near field. However, the radial variation in response of the poroelastic sample also has an influence on the magnitude of the sound field and therefore influences the sound field in the whole Kundt tube. This can be concluded from figure 3(a), since the measurement points used to calculate the absorption coefficient are not located in the near field. This figure also illustrates the ISO 10534-2 recommendation that the microphone should be located at at least one diameter $(8 \mathrm{~mm})$ distance from the sample, in order to recover an approximately planar wave sound field [30].

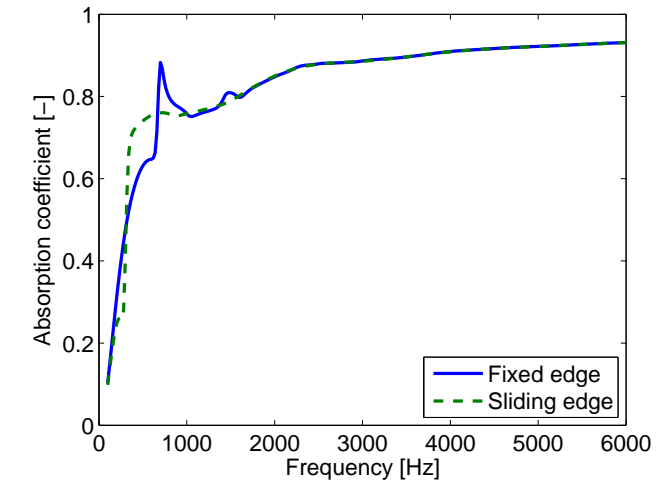

(a) Absorption curves for fixed edge and sliding edge conditions

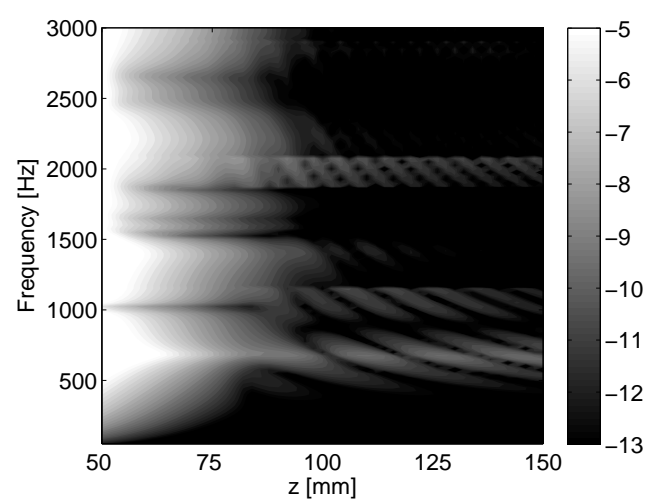

(b) Logarithm of the deviation of a 1D field $\hat{p}\left(z_{j}\right)$ as a function of the axial coordinate $z$.

Figure 3: Quantification of the radial variation of the sound field of eurocell.

One quantity to assess the effect of a poroelastic material on the sound field is the reflection coefficient. The definition of the reflection coefficient implicitly uses the assumption of a 1D surface wave and the existence of only one incident and one reflected wave. Therefore, the reflection coefficient may not be the best quantity for exploiting the 2D information the WBM model provides. In the ideal case and for a 1D model, the calculation of the reflection coefficient using (3) yields the same result for all couples of two measurement points. In practice however, the clamping conditions of the poroelastic sample cause 2D effects and consequently gives differences in the reflection coefficient depending on its location. Also, noise causes differences in reflection coefficients measured at different locations. This makes it useful to define different reflection coefficients, based on different measurement points as follows:

$$
R_{i}=\frac{e^{j k x_{1}}-e^{j k x_{i}} p_{1} / p_{i}}{e^{-j k x_{i}} p_{1} / p_{i}-e^{-j k x_{1}}}, i \neq 1,
$$

where $x_{i}$ is the distance of measurement point $i$ to the poroelastic sample and $p_{i}$ the pressure in this measurement point. The use of multiple reflection coefficients makes it possible to use more microphone locations in the Kundt tube, thus filtering out measurement noise and taking into account the radial variation of the sound field.

Another parameter which can be used for the quantification of the influence of the poroelastic material on the sound field is the relative pressure $p_{\text {rel }}$. This parameter uses the pressure measurements in a more direct way, without using any $1 \mathrm{D}$ assumptions and can also be calculated for different measurement points. Unlike the reflection coefficient, the relative pressure is, even in the $1 \mathrm{D}$ case, different for different measurement point combinations and is defined as:

$$
p_{r e l, i}=\frac{p_{1}^{2}+p_{i}^{2}}{p_{1} p_{i}}, i \neq 1,
$$

with $p_{i}$ the pressures at the different measurement points and $p_{1}$ the pressure at the reference point. 


\section{Sensitivity analysis}

A first step towards a material characterisation is performing a sensitivity analysis of the quantification parameters (reflection coefficient and relative pressure) to the primary material parameters. This sensitivity analysis gives more insight in the problem of parameter estimation for a number of reasons. First, a sensitivity analysis can show which material parameters have a large influence on the quantification parameters. Parameters with a small influence on the studied quantity will probably be harder to estimate through a model inversion, since a change in these parameters will only have a small effect on this quantity. Second, a sensitivity analysis can find the frequency dependency of these quantities. This knowledge can be used to determine the frequency range which is used for the parameter identification. Third, a sensitivity analysis can determine which spatial locations of the microphones are suited best to obtain a high sensitivity and thus probably a better parameter estimation performance.

The sensitivity analysis is performed by calculating the sensitivity for 100 frequencies, linearly divided in the range from $100 \mathrm{~Hz}$ to $3000 \mathrm{~Hz}$. For convenience and to lower the calculation time, the sensitivity analysis is carried out in two steps using the chain rule:

$$
S_{\lambda}=\frac{\partial H}{\partial \lambda}=\frac{\partial H}{\partial \chi_{R e}} \frac{\partial \chi_{R e}}{\partial \lambda}+\frac{\partial H}{\partial \chi_{I m}} \frac{\partial \chi_{I m}}{\partial \lambda}
$$

where $S_{\lambda}$ is the sensitivity to a material parameter, $H$ is the quantity of which the sensitivity is determined (e.g. the reflection coefficient), $\lambda$ is a material parameter (e.g. the tortuosity) and $\chi$ is an intermediary complex-valued parameter. While the influence of the material parameter $\lambda$ on the intermediary parameter can be determined analytically, the calculation of sensitivity of $H$ to this intermediary parameter $\chi$ needs the solution of the entire model of the Kundt tube. Therefore, the inversion of an ill-conditioned system of equations is necessary, which is resource intensive. By using the intermediary parameter $\chi$, the computational effort needed to perform the sensitivity analysis is vastly reduced. The intermediary parameters used in this paper are $K_{f}$ and $\tilde{\rho}_{11}$ and appear directly in the Biot equations. If the sensitivity of $H$ to a parameter $\lambda$ is influenced via the intermediary parameter $\tilde{\rho}_{11}$, it will also be influenced through $\tilde{\rho}_{12}$ and $\tilde{\rho}_{22}$. However, the influence on the sensitivity of these three parameters is very similar. Therefore it suffices to study the sensitivity of $H$ to $\lambda$ through only one of these parameters, $\tilde{\rho}_{11}$.

\subsection{Material dependency of the sensitivity}

Figure 4 shows the rms-average over all frequencies of the sensitivity of the reflection coefficient to the different material parameters for 5 materials, as specified in Appendix A. These materials have very different material parameters. Therefore, the analysis based on these 5 materials is representative for a general poroelastic material.

The sensitivity of the relative pressure is very similar and is not shown. From this figure follows the low sensitivity to $\Lambda$ and, less pronounced, to $N_{I m}$, which suggests that these parameters could cause difficulties in a parameter estimation. The former is to be expected as the samples are relatively thick and the flow resistivities not too high, such that the viscous effects are mainly driven by the flow resistivity. When the sensitivity to a given parameter $\lambda$ is low, differences in the value of this parameter lead to hardly different values of the quantification parameter $H$, which makes it difficult to detect differences in $\lambda$ using this quantification parameter.

\subsection{Frequency dependency of the sensitivity}

An analysis of the frequency dependency of the sensitivity of the quantity $H$ can show which frequency region is best suited for the estimation of the material parameters. As pointed out earlier, this is the frequency range in which the sensitivity to the material parameters is highest.

Figure 5 shows the frequency dependency of the sensitivity to $E_{1, I m}$ and $\Lambda^{\prime}$. Although the ratio of the sensitivities for the different materials differs strongly between the different material parameters, the frequency dependencies of the sensitivity to most other parameters are very similar to that of $E_{1, I m}$. Only the sensitivity to $\Lambda^{\prime}$ exhibits a strongly different frequency dependency, which is probably because $\Lambda^{\prime}$ is the only material parameter propagating through $\tilde{\rho}_{11}$. The other material parameters propagate through $\tilde{K}_{f}$.

Figure 5a shows that fireflex 1 , fireflex 2 , eurocell and urecom have a zone of high sensitivity at frequencies below $1500 \mathrm{~Hz}$, while melamine exhibits a zone of high sensitivity at higher frequencies. It can therefore be concluded that it is difficult to unambiguously define a frequency range to estimate the material parameters, since the region which exhibits a high sensitivity to the material parameters differs for each material. 


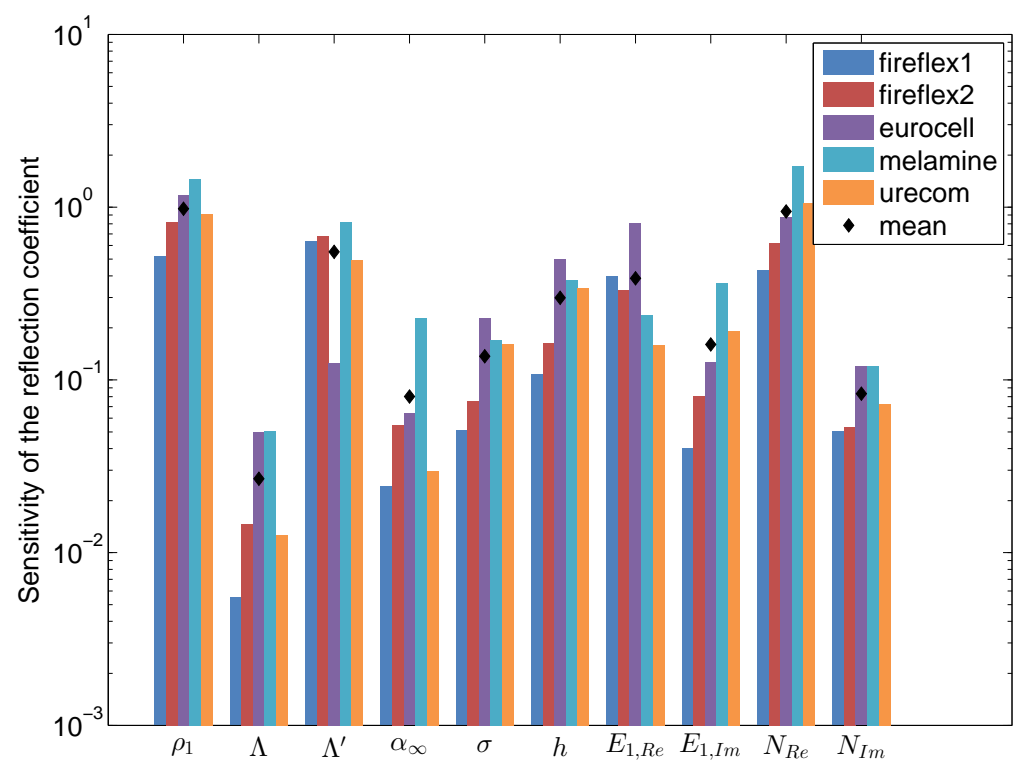

Figure 4: Sensitivity of the reflection coefficient and mean sensitivity of all materials, for a test setup as specified in section 2.2 and a sample thickness of $5 \mathrm{~mm}$.

The sample thickness has an influence on the frequency dependency of the sensitivity. On one hand, a change in thickness causes a change in resonance frequencies. On the other hand, the sensitivity to some material parameters is higher when using a thinner sample. This provides possibilities for enhancing the parameter estimation convergence, which are, however, not further discussed in this paper, as it is often not straightforwardly possible to obtain material samples with a different thickness.

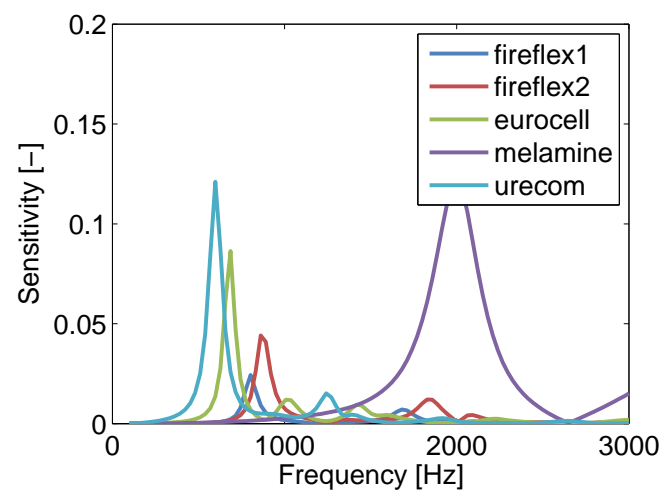

(a) $E_{1, I m}$

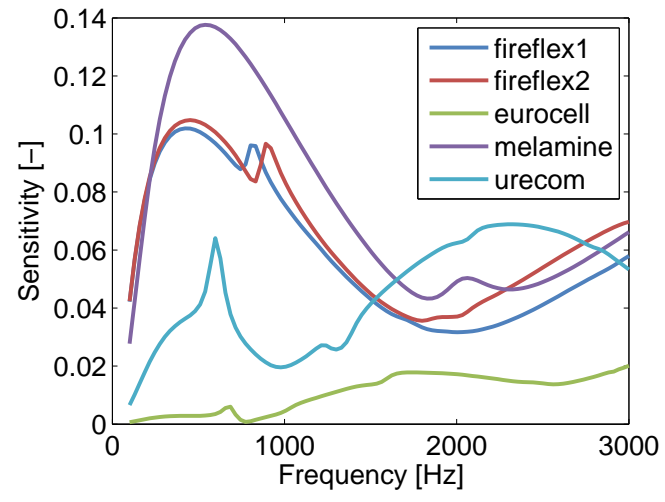

(b) $\Lambda^{\prime}$

Figure 5: Frequency dependency of the sensitivity of the reflection coefficient for the test setup as described in section 2.2.

\subsection{Spatial dependency of the sensitivity}

While the reflection coefficient would be the same for every location of the measurement points in the case of perfect $1 \mathrm{D}$ behaviour, the value of the relative pressure depends on the location of these measurement points. A 
sensitivity analysis can therefore be used to determine an optimal axial location for these points. The measurement point locations are constrained to the inner surface of the Kundt tube, just like in a real setup where they are positioned in order not to disturb the sound field. Figure 6(a) shows the spatial sensitivity of the relative pressure to the tortuosity $\alpha_{\infty}$ for melamine. The positions of the measurement points on the $\mathrm{x}$ - and $\mathrm{y}$-axis are measured in terms of the distance to the poroelastic sample. Since the definition of relative pressure is symmetric, only half of the domain for the sensitivity analysis needs to be calculated. Again, apart from $\Lambda^{\prime}$, the sensitivity of the other material parameters is very similar to that of $\alpha_{\infty}$.

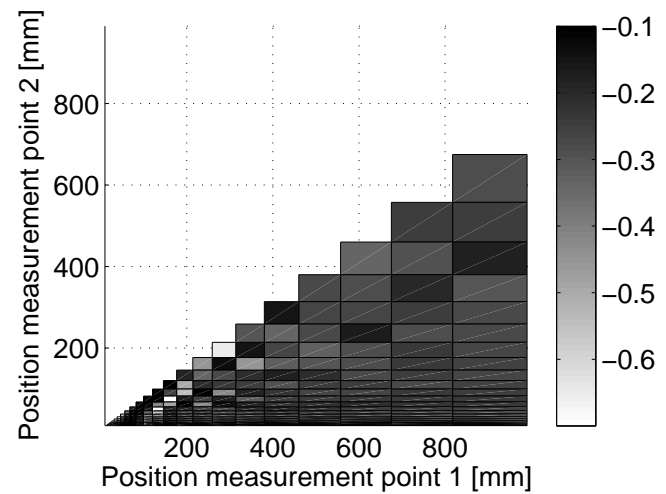

(a) $\alpha_{\infty}$

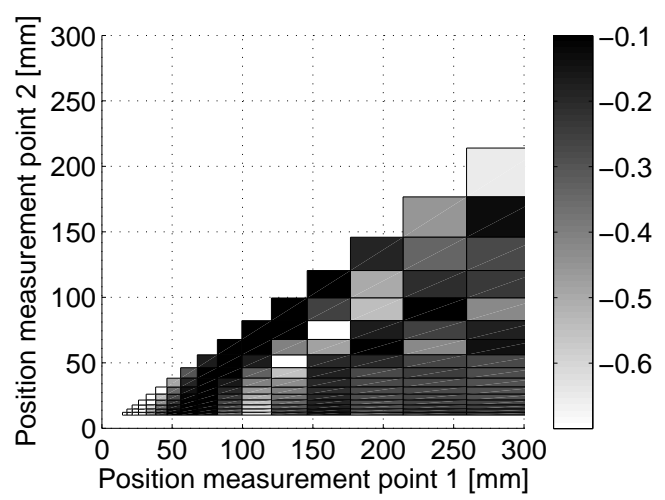

(b) $\alpha_{\infty}$ - detail

Figure 6: Logarithm of the sensitivity of the relative pressure for melamine.

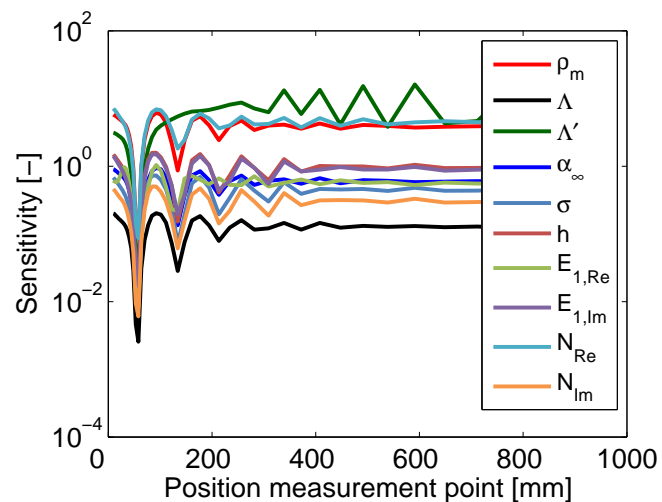

(a) melamine

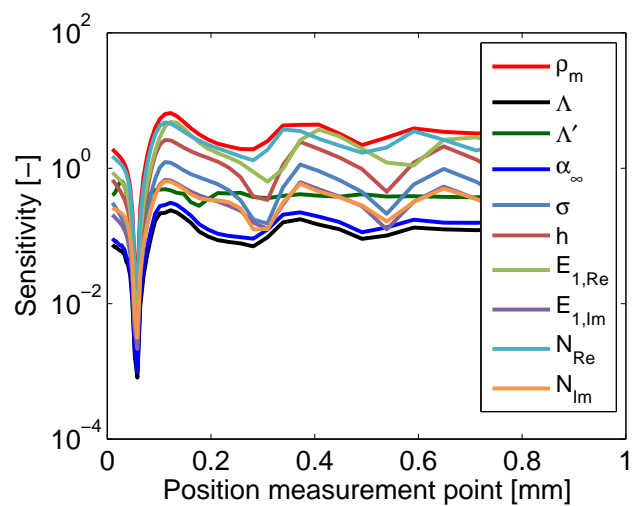

(b) eurocell

Figure 7: Sensitivity for the relative pressure using a reference pressure at $d_{1}=5.6 \mathrm{~cm}$ from the poroelastic material.

From these figures it is clear that (except for $\Lambda^{\prime}$ ) the sensitivity to the material parameters is the highest at measurement point locations close to the sample. Therefore, figure 6(b) shows a close-up of figure 6(a). This figure indicates that, rather than only one optimal pair of points, there exists a combination of point pairs with an approximately equally high sensitivity. To take near field information into account, a first point is chosen very close to the sample. A distance of $d_{2}=1 \mathrm{~cm}$ yields an optimal distance of $d_{1}=5.6 \mathrm{~cm}$ from the sample for the second point. This second point $d_{1}$ is taken as the reference point, since it contains less near-field information and therefore can be intuitively seen as a more robust reference.

Once this reference point is determined, additional measurement points can be found by performing a sensitivity analysis based on this reference point. Figure 7(a) shows the result of the sensitivity analysis for melamine. Based 
on this sensitivity analysis, a number of locations for additional measurement points is defined. Table 1 shows the different measurement point configurations used in this paper.

\begin{tabular}{ll}
\hline Number of measurement points & $\begin{array}{l}\text { Location of the measurement points } \\
\text { (distance to poroelastic sample in } \mathrm{mm} \text { ) }\end{array}$ \\
\hline 2 measurement points, original configuration & $89 ; 139$ \\
2 measurement points, optimised configuration & $56 ; 10$ \\
4 measurement points & $56 ; 10 ; 100 ; 180$ \\
7 measurement points & $56 ; 10 ; 100 ; 180 ; 260 ; 380 ; 650$ \\
\hline
\end{tabular}

Table 1: Location of measurement points for material characterisation based on the relative pressure.

However, figure 7(b) shows that for example for eurocell the sensitivity as a function of the location of extra measurement points is entirely different. Measurement points with a high sensitivity for one material will therefore not necessarily have a high sensitivity for another material. This makes it very difficult to determine a location which a priori optimises the parameter estimation for an unknown material. This paper uses the measurement points in Table 1, defined based on the sensitivity of melamine. Therefore, it can be expected that the parameter estimation procedure converges better for melamine using relative pressures, but not necessarily for the other materials. Since it is impossible to determine the optimal measurement point location before identifying the material, it is reasonable to define the measurement setup based on one given material.

\subsection{Conclusion}

As this section shows, the sensitivity of the quantification parameters is strongly dependent on the test sample itself, the frequency band considered and the location of the measurement points. Besides, these sensitivities depend strongly on the considered material. This makes it difficult to specify a generally applicable measurement test lay-out for the parameter identification of poroelastic materials. In this paper, the optimal measurement point locations for melamine were used. It is not trivial to optimise the measurement point locations for a broad range of materials. As a rule of thumb, one should locate at least one point in the near field to exploit the extra information the axisymmetric Kundt tube model offers over 1D models. Based on the sensitivity analysis, frequency ranges in which the parameter estimation is performed are also selected.

\section{Parameter estimation using global optimisation}

The goal of this paper is to assess whether the actual clamping conditions of a sample can be exploited for parameter estimation using global optimisation methods. To this goal, an efficient WBM model of the Kundt tube is used. The parameter estimation problem is formulated as a minimisation problem in which a goal function $f(\mathbf{x})$ is minimised. As Figure 8 shows, this goal function calculates the difference between the quantification parameter as calculated with the estimated material parameters and the measurements of the quantification parameter, in a least squares formulation. The quantification parameter can be the reflection coefficient or relative pressure. This paper only considers simulation-based measurements, generated by a forward simulation of the poroelastic Kundt tube model. Symbolically, the goal function at iteration $j$ of the optimisation procedure can be expressed as:

$$
f\left(\mathbf{x}_{\mathbf{j}}\right)=\left\|\mathbf{\Phi}\left(\mathbf{x}_{\mathbf{j}}\right)-\mathbf{\Phi}_{\mathbf{m}}\right\|_{2}^{2},
$$

where $\boldsymbol{\Phi}_{\mathbf{m}}$ is the quantification parameter calculated from the (virtual) measurements and $\boldsymbol{\Phi}\left(\mathbf{x}_{\mathbf{j}}\right)$ is the quantification parameter calculated from estimate $j$ of the material parameters using a forward simulation of the Kundt tube WB model. In this formulation the quantification parameters $\Phi\left(\mathbf{x}_{\mathbf{j}}\right)$ and $\boldsymbol{\Phi}_{\mathbf{m}}$ are expressed as vectors, with as entries the real and imaginary part (in the case of the relative pressure as the quantification parameter) for each measurement 
point:

$$
\boldsymbol{\Phi}\left(\mathbf{x}_{\mathbf{j}}\right)=\left[\begin{array}{c}
R_{1}\left(\mathbf{x}_{\mathbf{j}}\right) \\
\vdots \\
R_{i}\left(\mathbf{x}_{\mathbf{j}}\right) \\
\vdots \\
R_{N}\left(\mathbf{x}_{\mathbf{j}}\right)
\end{array}\right] \quad \text { or } \quad \boldsymbol{\Phi}\left(\mathbf{x}_{\mathbf{j}}\right)=\left[\begin{array}{c}
\operatorname{Im}\left(p_{\text {rel }, 1}\left(\mathbf{x}_{\mathbf{j}}\right)\right) \\
\operatorname{Re}\left(p_{\text {rel }, 1}\left(\mathbf{x}_{\mathbf{j}}\right)\right) \\
\vdots \\
\operatorname{Im}\left(p_{\text {rel, },}\left(\mathbf{x}_{\mathbf{j}}\right)\right) \\
\operatorname{Re}\left(p_{\text {rel, },}\left(\mathbf{x}_{\mathbf{j}}\right)\right) \\
\vdots \\
\operatorname{Im}\left(p_{\text {rel, }, N}\left(\mathbf{x}_{\mathbf{j}}\right)\right) \\
\operatorname{Re}\left(p_{\text {rel, }}\left(\mathbf{x}_{\mathbf{j}}\right)\right)
\end{array}\right],
$$

for reflection coefficients (7) and relative pressures (8), respectively. The quantification parameter $\boldsymbol{\Phi}_{\mathbf{m}}$ needs to be only calculated one time from the (virtual) pressure measurements, while $\boldsymbol{\Phi}\left(\mathbf{x}_{\mathbf{j}}\right)$ needs to be calculated in each iteration $j$ of the optimisation since the reflection coefficient and relative pressure are in that case a function of $\mathbf{x}_{\mathbf{j}}$. The optimisation variable $\mathbf{x}$ contains all the aforementioned material parameters. Therefore, the optimisation method used tries to estimate all material parameters simultaneously.

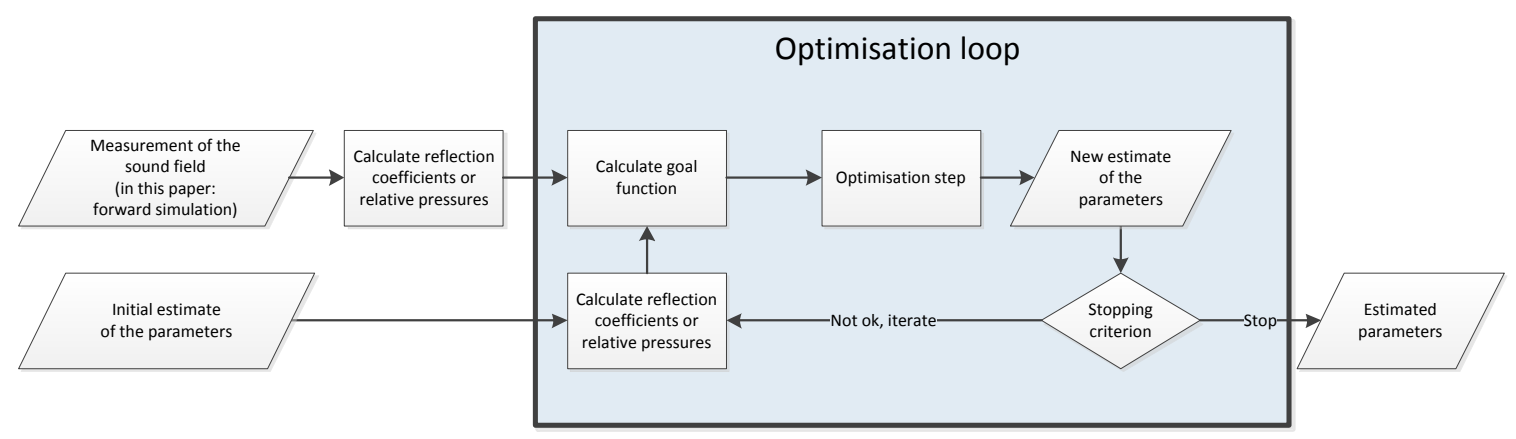

Figure 8: Flowchart of the general optimisation procedure.

Most commonly, local optimisation methods are used for the minimisation of the goal function. These methods start from an initial estimate of the optimisation variable $\mathbf{x}$, and try to find in each iteration a vector $\mathbf{x}$ which leads to a lower value of the goal function. When one of the stopping criteria is met, the last optimisation variable value is taken as the optimal one. An example of a stopping criterion is that value of the goal function or the optimisation variable changes less than a specified tolerance.

Optimisation problems are in general formulated in the following way, with $g(\mathbf{x})$ and $h(\mathbf{x})$ the equality and inequality constraints respectively [31]:

$$
\begin{array}{cl}
\underset{\mathbf{x} \in \mathbb{R}^{n}}{\operatorname{minimise}} & f(\mathbf{x}) \\
\text { subject to } & g(\mathbf{x})=0, \\
& h(\mathbf{x}) \leq 0 .
\end{array}
$$

In the parameter estimation procedure discussed in this paper, no equality or inequality constraints are added, which leads to an unconstrained least-squares optimisation problem

Most optimisation routines use Newton-like (derivative-based) optimisation methods. For these methods, convergence is guaranteed for convex optimisation problems. In a convex optimisation problem, $f(\mathbf{x})$ and $h(\mathbf{x})$ in $(12)$ should be convex, while $g(\mathbf{x})$ should be affine [31]. The parameter estimation using the WBM model of the Kundt tube leads to a strongly non-convex problem. For such a problem, local optimisation does not necessarily lead to the 
global minimum of the goal function. In this case, the optimisation gets stuck in a local minimum of the goal function. In general, for a non-convex function it is impossible to verify if the attained local minimum is also the global one. Global optimisation methods try to cope with this problem, mostly by determining several local optima from different initial values for $\mathbf{x}$. The higher the number of initial values for $\mathbf{x}$, the higher the chances of convergence to the global optimum.

In a first step, no measurement noise is added to the simulated Kundt tube measurements. This makes it possible that the parameter estimation converges to the exact parameters.

\subsection{Multistart}

A first parameter estimation scheme uses MultiStart, a routine of the MATLAB Global optimisation Toolbox. This global optimisation solver starts a local optimiser at a number of starting points [32]. These starting points are generated using a random number generator. As a local optimiser, the non-linear least square solver lsqnonlin is used. This solver uses a trust region Newton-like method to locally solve the optimisation problem [33]. Figure 9 illustrates this procedure.

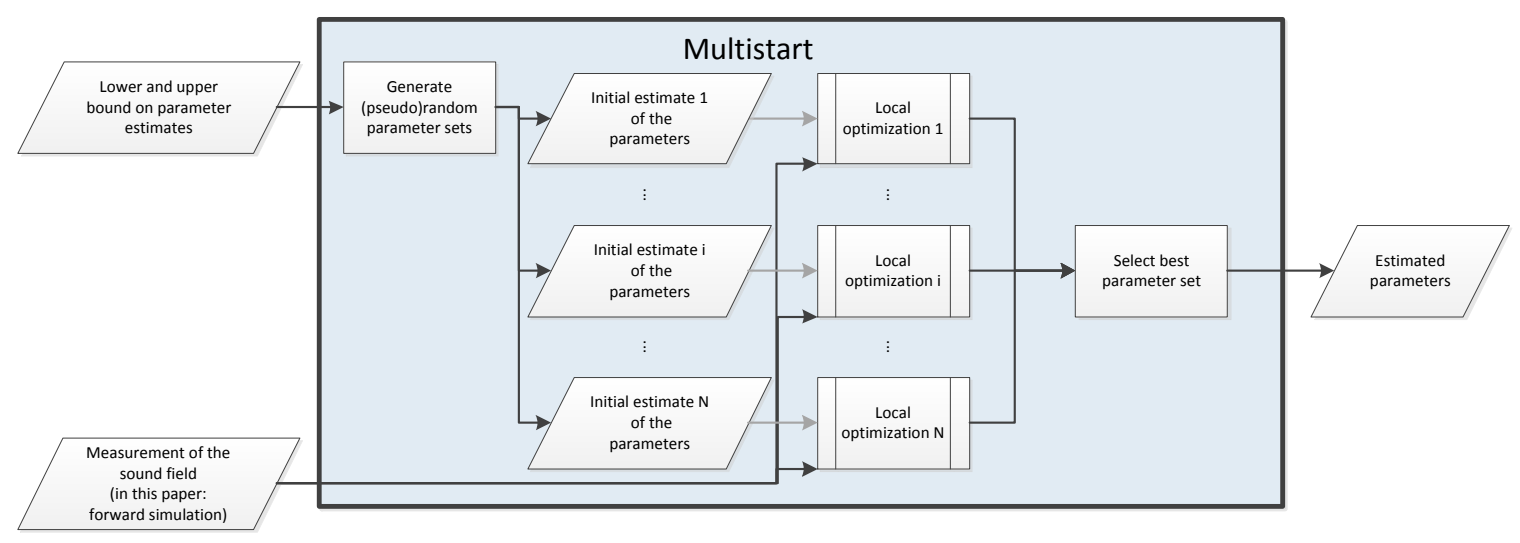

Figure 9: Flowchart of the Multistart global optimisation procedure.

\subsubsection{Procedure}

Section 4.2 points out that the sensitivity of both the reflection coefficient and the relative pressure to material parameters is strongly frequency dependent. Since these quantities are used to quantify the effect of the poroelastic material on the sound field, the performance of the parameter estimation will depend on the frequency range in which the estimation is performed. Based on figure 5(a) two frequency ranges are determined, containing 100 frequencies each, linearly divided between a minimum and maximum value. A first range includes frequencies from $100 \mathrm{~Hz}$ up to $1500 \mathrm{~Hz}$, since certain materials (like fireflex 1, fireflex 2, eurocell and urecom) exhibit a high sensitivity in this frequency region. A second frequency range includes frequencies from $100 \mathrm{~Hz}$ up to $3000 \mathrm{~Hz}$, since other materials (e.g. melamine) show a high sensivity only above $1500 \mathrm{~Hz}$.

The local 1sqnonlin solver stops at the following stopping criteria: (i) the 2-norm of the difference of 2 consecutive iterations of the optimisation variable is less than $10^{-5}$; (ii) the difference in goal function of 2 consecutive iterations is less than $10^{-12}$; (iii) the number of goal function evaluations exceeds 333 (for the frequency range up to $3000 \mathrm{~Hz}$ ). The optimisation in the frequency range up to $1500 \mathrm{~Hz}$ uses as stopping condition (iii) a maximum of 441 goal function evaluations, since the data for this case is taken from a MultiStart-run performed as part of a further described multi-step procedure. The MultiStart parameter estimation is performed for different test setup configurations, as defined in Table 1, based on a sensitivity study. The optimisation is first performed for all materials described in Appendix A using 5 starting points. In order to always start from the same starting points, the random 
number generator used to generate these points is reset before each simulation. For melamine, a material which exhibits a low computational cost, the parameter estimation is also started from 40 starting points.

\subsubsection{Results}

Table 2 shows how many out of 5 parameter estimations converge to the correct material parameters. If the parameters could not be estimated, the number of convergences is grayed out. In the frequency range up to 1500 $\mathrm{Hz}$, the parameters are correctly estimated for all materials except for melamine. However, the estimations using frequencies up to $3000 \mathrm{~Hz}$ do not succeed in finding the correct parameters for any material, exept for melamine. This is consistent with a good parameter estimation in the frequency ranges with a high sensitivity, as shown on figure 5(a). Indeed, melamine exhibits the highest sensitivity between $1500 \mathrm{~Hz}$ and $3000 \mathrm{~Hz}$, while the other materials show a high sensitivity at lower frequencies. It can therefore be concluded that a high sensitivity in the frequency range used leads indeed to a better convergence behaviour. Table 2 also shows that the parameter estimation converges in at least one of both frequency ranges.

\begin{tabular}{|c|c|c|c|c|}
\hline Material & $\begin{array}{c}1500 \mathrm{~Hz} \\
\text { Relative pressure, } \\
7 \text { measurement points }\end{array}$ & $\begin{array}{l}\qquad 3000 \mathrm{~Hz} \\
\text { Reflection coefficient, } \\
\text { original configuration }\end{array}$ & $\begin{array}{c}3000 \mathrm{~Hz} \\
\text { Relative pressure, } 4 \\
\text { measurement points }\end{array}$ & $\begin{array}{l}\text { } 3000 \mathrm{~Hz} \\
\text { Relative pressure, } 7 \\
\text { measurement points }\end{array}$ \\
\hline Fireflex1 & $3 / 5$ & $0 / 5$ & $0 / 5$ & $0 / 5$ \\
\hline Fireflex 2 & $2 / 5$ & $0 / 5$ & $0 / 5$ & $0 / 5$ \\
\hline Eurocell & $5 / 5$ & $0 / 5$ & $0 / 5$ & $0 / 5$ \\
\hline Melamine & $0 / 5$ & $2 / 5$ & $2 / 5$ & $2 / 5$ \\
\hline Urecom & $2 / 5$ & $0 / 5$ & $0 / 5$ & $0 / 5$ \\
\hline
\end{tabular}

Table 2: Convergence of the parameter estimations based on 5 starting points in MultiStart.

When increasing the number of points to 40 points for melamine, it shows that the parameter estimation using the reflection coefficient performs better than the estimation using relative pressures based on 7 measurement points, as Table 3 shows. The parameter estimation using 4 measurement points for the relative pressure performs best. In general, the percentage of converging parameter estimations is similar to the results obtained in Table 2.

\begin{tabular}{lccc}
\hline Material & $\begin{array}{l}\text { Reflection coefficient, } \\
\text { original configuration }\end{array}$ & $\begin{array}{l}\text { Relative pressure, } \\
4 \text { measurement points }\end{array}$ & $\begin{array}{l}\text { Relative pressure, } \\
7 \text { measurement points }\end{array}$ \\
\hline Melamine & $19 / 40$ & $21 / 40$ & $16 / 40$ \\
\hline
\end{tabular}

Table 3: Convergence of the parameter estimation based on 40 starting points in MultiStart, using frequencies up to $3000 \mathrm{~Hz}$.

\subsubsection{Conclusion}

The parameter estimation using MultiStart leads to two observations. First, the highly frequency-dependent sensitivity of both reflection coefficient and relative pressure is different for different materials. Section 5.1.2 pointed out that this difference also causes a difference in convergence to the correct parameters. Therefore, it is very difficult to define a frequency domain in which the optimisation converges to the correct parameters for every material. Second, when the parameter estimation does not converge to the correct parameters, always the same parameters have a large relative error as compared to the correct ones. In the case of the parameter estimation using frequencies up to $1500 \mathrm{~Hz}$, the parameters which show the largest relative error are $E_{1, I m}, N_{I m}$ and $E_{1, R e}$. The difference in convergence behaviour between reflection coefficient and relative pressures is not large enough to draw any conclusions to that respect.

\subsection{3-step procedure}

The previous section shows it is not possible to define one frequency domain which gives good estimation results for all materials, and that always the same parameters do not converge well. A 3-step procedure can lead to better performance by solving these problems. On the one hand, it combines a parameter estimation using frequencies up to $1500 \mathrm{~Hz}$ and an estimation using frequencies up to $3000 \mathrm{~Hz}$, in order to perform the parameter estimation in two different frequency ranges. On the other hand, this 3-step procedure creates a lot of different starting points for the parameters $E_{1, I m}, N_{I m}$ and $E_{1, R e}$, because these are the parameters showing the largest error when optimising using 
MultiStart. By starting from a lot of starting points for this parameters, the probability of converging to the right value for these parameters is higher.

\subsubsection{Procedure}

The 3-step procedure, as shown by Figure 10 is a novel procedure which tries to find all material parameters in 3 steps. In a first step, MultiStart performs a parameter estimation using frequencies up to $1500 \mathrm{~Hz}$ starting from $N_{1}$ starting points. The second step creates a grid for $E_{1, I m}, N_{I m}$ and $E_{1, R e}$. For these parameters, the grid contains $N_{2}$ equally spaced points between certain boundaries, specified below. For the other parameters, all grid points contain the parameter value of the parameter estimation with the lowest goal function in the first step. This second step also evaluates the goal function value of all these grid points. The third step of the 3-step procedure starts a second MultiStart optimisation which uses the $N_{3}$ grid points with the lowest goal function obtained in step 2 as starting points. This parameter estimation uses frequencies up to $3000 \mathrm{~Hz}$.

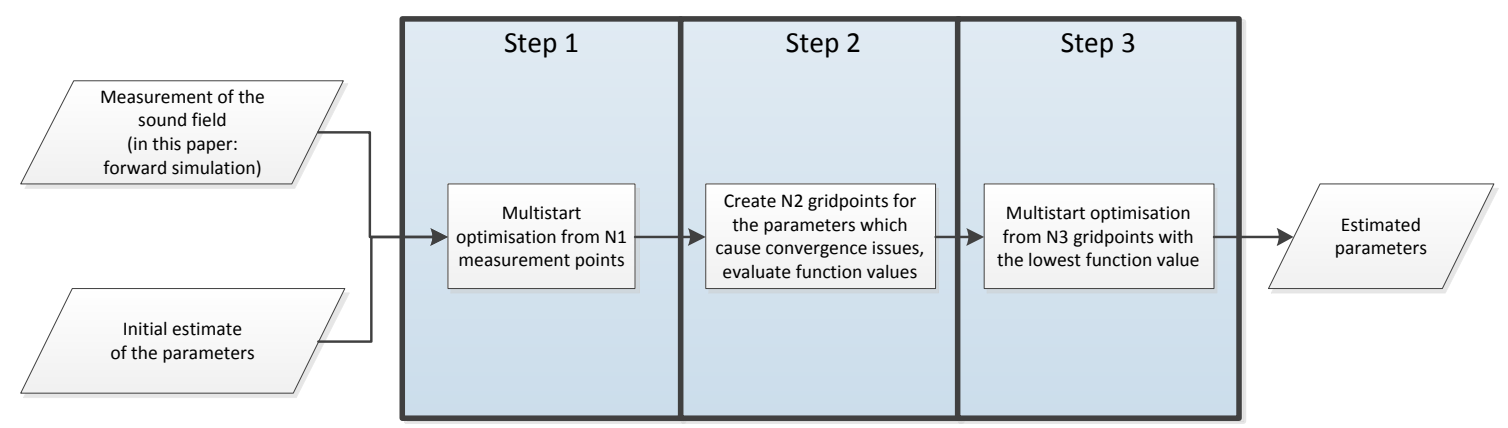

Figure 10: Flowchart of the 3-step global optimisation procedure.

The 3-step procedure uses for Multistart the same stopping criteria as described before, with a maximum of 441 function evaluations (criterion (iii)). The parameters of the 3 step procedure are set as follows: $N_{1}=8, N_{2}=5$ and $N_{3}=4$. To speed up the parameter estimation, the 3-step procedure skips the second and third step if the goal function value is already smaller than $f_{\text {conv }}=10^{-9}$.

The lower boundaries to construct the grid for $E_{1, R e}, E_{1, I m}$ and $N_{I m}$ are respectively $50 \cdot 10^{3} \mathrm{~Pa}, 2.5 \cdot 10^{3} \mathrm{~Pa}$ and $2 \cdot 10^{3} \mathrm{~Pa}$. The upper boundaries for these parameters are, in the same order, $500 \cdot 10^{3} \mathrm{~Pa}, 200 \cdot 10^{3} \mathrm{~Pa}$ and $50 \cdot 10^{3} \mathrm{~Pa}$. These boundaries are based on the extreme values of all materials. Since this range is only used for the determination of the starting points for the optimisations and not used as a constraint during the optimisation, the correct parameters of a material can still be estimated, even if these parameters would lie outside these boundaries.

\subsubsection{Results}

The 3-step procedure is applied for different measurement point configurations for both reflection coefficient and relative pressure. Tables 4 and 5 show how many of the optimisations (using 4 or 8 different starting points) converge to the correct parameter estimation for reflection coefficient and relative pressure, respectively.

A comparison of the results in both tables shows clearly that the reflection coefficient-based estimations lead to a more robust parameter estimation than the relative pressure-based ones. The reflection coefficients lead to a correct estimation of all material parameters in all cases, except if 7 measurement points are used. However, the parameter estimation using relative pressures fails to estimate the parameters of different materials in most cases. The cases where none of the starting points in step 1 and 3 leads to a convergence to the right material parameters are grayed out in Tables 4 and 5. For example, the parameters of fireflexl are correctly estimated in the reflection coefficient optimisations (first line of Table 4), but not in all cases when using relative pressures (first line of Table 5). As shown by (7), reflection coefficients take the microphone position relative to the poroelastic sample explicitly into account. 
Even though this analytical expression only holds in a 1D field, this additional information is a plausible explanation for the better convergence of parameter estimations using reflection coefficients.

The influence of the number of measurement points and their location is less pronounced. This can be explained by noting that the optimal measurement point location is different for different materials. Therefore, the used measurement points, which are optimised for melamine, may deteriorate the performance of the parameter estimation for other materials. Indeed, the sensitivity of additional measurement points can be lower than the sensitivity of the existing ones, and may thus have a negative influence on the convergence. In general, it can be concluded that there is no identifiable advantage in using different measurement points as the original measurement setup.

\begin{tabular}{|c|c|c|c|c|c|c|c|c|}
\hline \multirow[t]{2}{*}{ Material } & \multicolumn{2}{|c|}{ Original measurement points } & \multicolumn{2}{|c|}{2 measurement points } & \multicolumn{2}{|c|}{4 measurement points } & \multicolumn{2}{|c|}{7 measurement points } \\
\hline & Step 1 & Step 3 & Step 1 & Step 3 & Step 1 & Step 3 & Step 1 & Step 3 \\
\hline Fireflex1 & $2 / 8$ & - & $1 / 8$ & - & $2 / 8$ & - & $1 / 8$ & - \\
\hline Fireflex2 & $3 / 8$ & - & $2 / 8$ & - & $3 / 8$ & - & $2 / 8$ & - \\
\hline Eurocell & $2 / 8$ & - & $6 / 8$ & - & $4 / 8$ & - & $5 / 8$ & - \\
\hline Melamine & $0 / 8$ & $1 / 4$ & $0 / 8$ & $3 / 4$ & $0 / 8$ & $2 / 4$ & $0 / 8$ & $0 / 4$ \\
\hline Urecom & $4 / 8$ & - & $2 / 8$ & - & $2 / 8$ & - & $2 / 8$ & - \\
\hline
\end{tabular}

Table 4: Convergence of the parameter estimation using the 3-step procedure, reflection coefficient.

\begin{tabular}{l|cc|cc|ccc|c}
\hline Material & \multicolumn{2}{|c|}{ Original measurement points } & \multicolumn{2}{|c|}{ 2 measurement points } & \multicolumn{2}{c}{4 measurement points } & \multicolumn{2}{c}{7 measurement points } \\
& Step 1 & Step 3 & Step 1 & Step 3 & Step 1 & Step 3 & Step 1 \\
\hline Fireflex1 & $0 / 8$ & $0 / 4$ & $0 / 8$ & $0 / 4$ & $0 / 8$ & $2 / 4$ & $2 / 8$ \\
Fireflex2 & $3 / 8$ & - & $2 / 8$ & - & $3 / 8$ & $3 / 4$ & $1 / 8$ \\
Eurocell & $3 / 8$ & - & $0 / 8$ & $0 / 4$ & $4 / 8$ & $4 / 4$ & $5 / 8$ \\
Melamine & $0 / 8$ & $3 / 4$ & $0 / 8$ & $3 / 4$ & $0 / 8$ & $0 / 4$ & $0 / 8$ \\
Urecom & $5 / 8$ & - & $1 / 8$ & - & $2 / 8$ & $2 / 4$ & $1 / 8$ \\
\hline
\end{tabular}

Table 5: Convergence of the parameter estimation using the 3-step procedure, relative pressure.

\section{Effect of noise}

To verify the robustness of the parameter estimation, it is important to assess the effect of noise. Therefore Gaussian noise is added to the virtual measurements, generated by a forward simulation of the Kundt tube model, used in this section.

\subsection{Procedure}

The noise on the measurements, simulated by the Kundt tube model, is added by using the MATLAB routine randn with a standard deviation equal to the noise amplitude. According to literature, microphones suffer from a thermal noise of about $41 \mathrm{dBA}$ [34]. Thermal noise has a constant spectral power density and its amplitude has a Gaussian distribution $[35,36]$. In a first approximation, noise with an amplitude of $40 \mathrm{~dB}$ is therefore applied on the simulated measurements for all frequencies. This amplitude is equal to

$$
p=p_{\text {ref }} \cdot 10^{(40 / 20)} \approx 0.0020 \mathrm{~Pa}
$$

with $p_{\text {ref }}=2 \cdot 10^{-5} \mathrm{~Pa}$. The parameter estimation uses the 3-step procedure described in the previous section with the same parameters: $N_{1}=8, N_{2}=5$ and $N_{3}=4$.

Higher noise levels, as shown in Table 6 are applied on the simulated measurement results as well, to see at which point the parameter estimation does not converge any more. The parameter estimation is again performed for different measurement point locations, in order to assess the effect of these different configurations on the robustness of the parameter estimation. 


\subsection{Results}

Tables 6 and 7 show the results of the parameter estimation including measurement noise, when using the reflection coefficient and different measurement points for fireflex 2 and melamine. These two materials were chosen since they have a very different behaviour and are therefore representative for a large range of materials. These tables show for the first and third step of the 3-step procedure how many of the local solvers converge to the correct parameter values. Since the correct material parameters are known in this paper, it is easy to verify the convergence of the optimisation to the right solution. However, in practice it is difficult to assess if a found solution is a global one. The value of the goal function, the kind of stopping criterion which stopped the simulation (e.g. no more changes in the function value as compared to stopping after the maximum number of iterations) and comparison of different local optima can give an indication on whether the optimisation converged to the right parameters. For the parameter estimation using measurements including noise, the third step of the 3-step procedure is never skipped, because, due to the noise, the goal function value becomes never smaller than $f_{\text {conv }}=10^{-9}$.

These tables also illustrate that the effect of noise on the convergence is rather small. The number of convergences can even increase with added noise, for example for melamine, using the original Kundt tube setup. This can be explained by the influence of chance on the optimisation. Depending on the starting point, a local optimisation can evolve to the right parameters (global minimum) or get stuck in a local minimum. Since the addition of noise deforms the optimisation space, it is possible that more starting points converge to the global solution (change of the regions of attraction). While at a noise level of $80 \mathrm{~dB}$ most parameter estimations still converge to the right solution, in most cases the parameter estimation does not converge anymore when the noise level is $90 \mathrm{~dB}$.

\begin{tabular}{l|cc|cc|cc|c}
\hline & \multicolumn{2}{|c|}{ Original measurement points } & \multicolumn{2}{|c|}{ 2 measurement points } & \multicolumn{2}{|c|}{ 4 measurement points } & \multicolumn{2}{c}{7 measurement points } \\
& Step 1 & Step 3 & Step 1 & Step 3 & Step 1 & Step 3 & Step 1 \\
\hline No noise & $3 / 8$ & - & $2 / 8$ & - & $3 / 8$ & - & $2 / 8$ \\
$40 \mathrm{~dB}$ & $2 / 8$ & $1 / 4$ & $2 / 8$ & $0 / 4$ & $3 / 8$ & $1 / 4$ & $2 / 8$ \\
$50 \mathrm{~dB}$ & $4 / 8$ & $1 / 4$ & $2 / 8$ & $0 / 4$ & $3 / 8$ & $1 / 4$ & $2 / 8$ \\
$60 \mathrm{~dB}$ & $2 / 8$ & $1 / 4$ & $2 / 8$ & $0 / 4$ & $2 / 8$ & $0 / 4$ & $1 / 4$ \\
$70 \mathrm{~dB}$ & $2 / 8$ & $1 / 4$ & $2 / 8$ & $0 / 4$ & $1 / 8$ & $0 / 4$ & $1 / 4$ \\
$80 \mathrm{~dB}$ & $2 / 8$ & $1 / 4$ & $2 / 8$ & $1 / 4$ & $2 / 8$ & $1 / 4$ & $1 / 8$ \\
$90 \mathrm{~dB}$ & $0 / 8$ & $1 / 4$ & $0 / 8$ & $0 / 4$ & $0 / 8$ & $0 / 4$ & $2 / 8$ \\
\hline
\end{tabular}

Table 6: Convergence of the parameter estimation using the 3-step procedure and reflection coefficient, for measurements containing noise, fireflex2.

\begin{tabular}{l|cc|cc|cc|cc}
\hline & \multicolumn{2}{|c|}{ Original measurement points } & \multicolumn{2}{|c|}{2 measurement points } & \multicolumn{2}{|c|}{4 measurement points } & \multicolumn{2}{c}{7 measurement points } \\
& Step 1 & Step 3 & Step 1 & Step 3 & Step 1 & Step 3 & Step 1 & Step 3 \\
\hline No noise & $0 / 8$ & $1 / 4$ & $0 / 8$ & $3 / 4$ & $0 / 8$ & $2 / 4$ & $0 / 8$ \\
$40 \mathrm{~dB}$ & $0 / 8$ & $2 / 4$ & $0 / 8$ & $1 / 4$ & $0 / 8$ & $3 / 4$ & $0 / 8$ \\
$50 \mathrm{~dB}$ & $0 / 8$ & $2 / 4$ & $0 / 8$ & $3 / 4$ & $0 / 8$ & $3 / 4$ & $0 / 8$ \\
$60 \mathrm{~dB}$ & $0 / 8$ & $2 / 4$ & $0 / 8$ & $3 / 4$ & $0 / 8$ & $0 / 4$ & $3 / 4$ \\
$70 \mathrm{~dB}$ & $0 / 8$ & $2 / 4$ & $0 / 8$ & $0 / 4$ & $0 / 8$ & $0 / 8$ & $0 / 8$ \\
$80 \mathrm{~dB}$ & $0 / 8$ & $3 / 4$ & $0 / 8$ & $3 / 4$ & $0 / 8$ & $0 / 4$ & $0 / 8$ \\
$90 \mathrm{~dB}$ & $0 / 8$ & $2 / 4$ & $0 / 8$ & $0 / 4$ & $0 / 8$ & $0 / 4$ & $0 / 8$ \\
\hline
\end{tabular}

Table 7: Convergence of the parameter estimation using the 3-step procedure and reflection coefficient, for measurements containing noise, melamine.

Figures 11 and 12 show the maximum relative error on the material parameters for fireflex and melamine at different noise levels. This figure shows that in most cases, parameter estimations using the original measurement points yields smaller relative errors as compared to parameter estimations using other measurement point locations. The use of near field information or more measurement points seems to lead to a lower accuracy instead of a higher one. This can possibly be explained by the fact that the sound pressure is lower at the microphones located close to the poroelastic sample, which leads to a higher relative influence of the noise level on the quantification parameter. In general, it can be concluded that the optimisation procedure is very robust against noise: even when $80 \mathrm{~dB}$ of noise is added, the relative error on the estimated parameters is less than $10 \%$ when using the original measurement point location. 


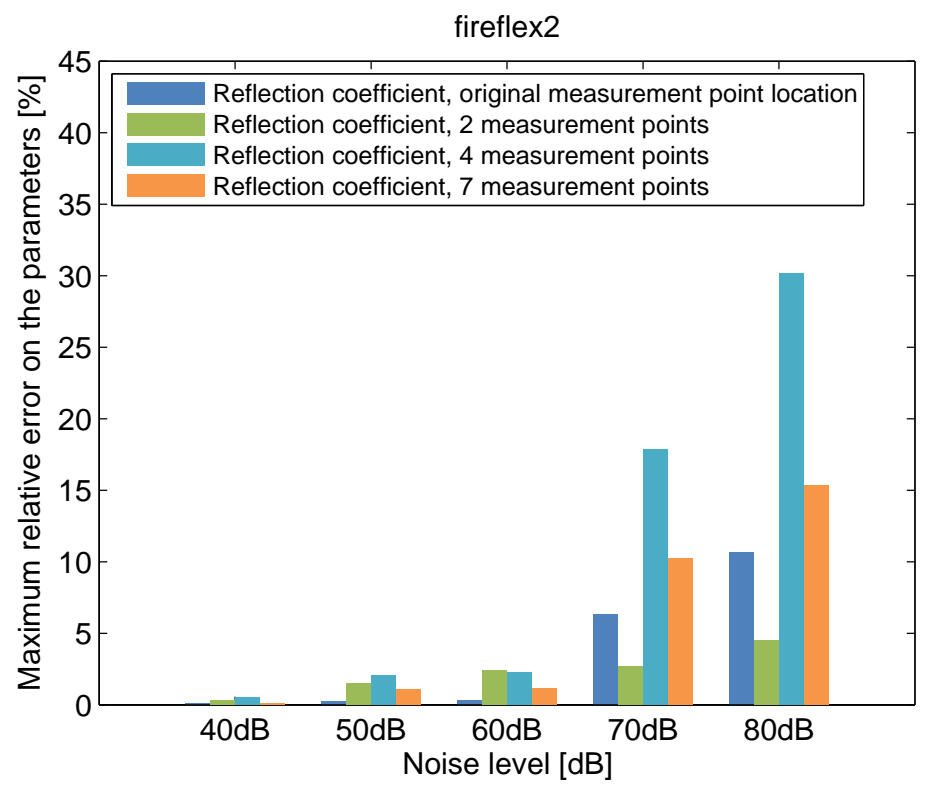

Figure 11: Maximum relative error on the material parameters for the parameter estimations using the 3-step procedure with measurements containing noise, for fireflex 2 .

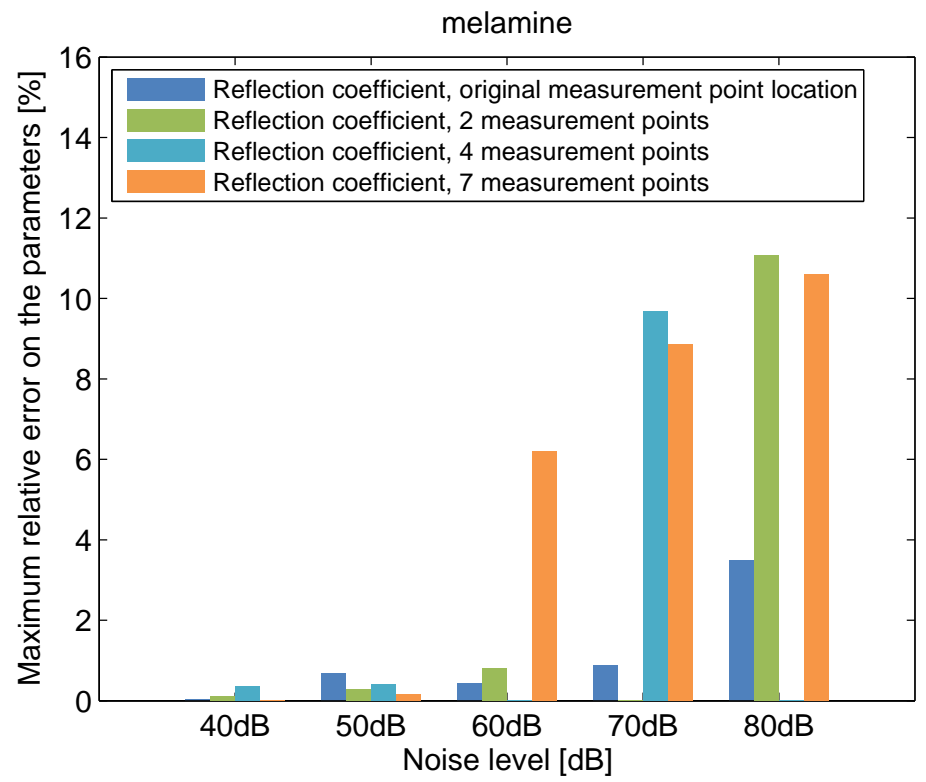

Figure 12: Maximum relative error on the material parameters for the parameter estimations using the 3-step procedure with measurements containing noise, for melamine.

\section{Conclusions}

This paper presents a method to characterise poroelastic materials using Kundt tube measurements in combination with efficient numerical WB models. The method developed assumes fixed edge boundary conditions, which means the poroelastic sample is clamped in the Kundt tube. Therefore, this paper assumes the boundary conditions are known. 
Two quantities taking into account the effect of the poroelastic material on the surrounding acoustic field are chosen: the reflection coefficient and the relative pressure. A sensitivity analysis studies the sensitivity of these 2 quantities to the material parameters. This analysis shows that $\Lambda$ and $N_{I m}$ are the parameters to which both quantities have the lowest sensitivity. The sensitivity analysis also demonstrates the strong frequency dependency of the sensitivity. Moreover, the sensitivity of the relative pressure depends on the measurement point locations. Besides, both frequency and spatial dependencies differ strongly for the different materials. Whereas parameter estimation methods can adapt to the difference related to the frequency dependency by using different frequency ranges, using different measurement points for different estimations is not feasible in practice.

Because of the strong non-convex character of the parameter estimation optimisation problem, local optimisation solvers do not converge to the correct material parameters. Therefore, this paper focuses on the use of global optimisation methods. These methods start an optimisation from pseudorandomly chosen starting points and therefore exhibit a higher chance of converging to the global minimum. Use of the MultiStart global optimisation method leads to the estimation of the correct material parameters for all materials either using a frequency range containing frequencies up to $1500 \mathrm{~Hz}$ or a frequency range containing frequencies up to $3000 \mathrm{~Hz}$. The 3-step procedure uses this insight to develop a more robust procedure. This procedure combines both frequency ranges and gives special attention to the parameters which do not converge well using MultiStart. This 3-step procedure finds the correct parameters for all materials. In general, the use of reflection coefficients leads to a more robust parameter estimation than using relative pressures. The reflection coefficient takes the distance of the microphones to the poroelastic sample explicitly into account, which is a possible explanation for this behaviour. There is no clearly identified advantage in the use of multiple measurement points or optimised measurement point configurations.

The effect of noise is investigated for the parameter estimation using reflection coefficients. A parameter estimation including (numerically generated) measurement noise points out that the influence of noise is rather small. The method used appears to be quite robust to noise, since even higher levels of noise (up to $80 \mathrm{~dB}$ for most measurement point configurations) do not lead to a worse convergence. Also this analysis incorporating noise does not show advantages of using the optimised measurement point configuration over the original one. To the contrary, the original measurement point configuration leads to a lower relative error on the parameters estimated. For this configuration, the relative error on the estimated parameters is smaller than $10 \%$ when $80 \mathrm{~dB}$ of noise is added. This illustrates the robustness of the developed procedure against noise. In general, it can therefore be concluded that the developed procedure leads to a robust parameter estimation for a broad range of materials, using a standard Kundt tube.

\section{Acknowledgements}

Elke Deckers is a postdoctoral fellow of the Fund for Scientific Research Flanders (F.W.O.). The institute for Promotion of Innovation by Science and Technology Flanders (Belgium) (IWT-Vlaanderen) is gratefully acknowledged for the support of the doctoral research of Stijn Jonckheere. Furthermore, the Research Fund KU Leuven is also gratefully acknowledged for its support.

\section{Appendix A. Material properties}

\begin{tabular}{ll}
\hline Absolute temperature & $T_{a b s}=297.45 \mathrm{~K}$ \\
Density & $\rho_{0}=1.1949 \mathrm{~kg} / \mathrm{m}^{3}$ \\
Dynamic viscosity & $\eta=15.5099 \cdot 10^{-6} \mathrm{~Pa} \cdot \mathrm{s}$ \\
Ratio of specific heats & $\gamma=1.4$ \\
Universal gas constant & $R=286.7 \mathrm{~J} /(\mathrm{kg} \cdot \mathrm{K})$ \\
Thermal conductivity & $\kappa=2.60 \cdot 10^{-2} \mathrm{~W} /(\mathrm{m} \cdot \mathrm{K})$ \\
Specific heat capapcity at constant pressure & $c_{p}=1005 \mathrm{~J} / \mathrm{kg} \cdot \mathrm{K}$ \\
\hline
\end{tabular}

Table A.8: Properties of air. 


\begin{tabular}{ll}
\hline Porosity & $h=0.95$ \\
Bulk density of the frame & $\rho_{1}=37.3 \mathrm{~kg} / \mathrm{m}^{3}$ \\
Tortuosity & $\alpha_{\infty}=1.17$ \\
Flow resistance & $\sigma=9200 \mathrm{~kg} /\left(\mathrm{m}^{3} \cdot \mathrm{s}\right)$ \\
Viscous characteristic length & $\Lambda=179 \cdot 10^{-6} \mathrm{~m}$ \\
Thermal characteristic length & $\Lambda^{\prime}=359 \cdot 10^{-6} \mathrm{~m}$ \\
Shear modulus of the frame & $N=66 \cdot 10^{3}+4 \cdot 10^{3} j \mathrm{~Pa}$ \\
Young's modulus of the frame in vacuum & $E_{1}=148 \cdot 10^{3}+14 \cdot 10^{3} j \mathrm{~Pa}$ \\
\hline
\end{tabular}

Table A.9: Properties of fireflex1.

\begin{tabular}{ll} 
Porosity & $h=0.95$ \\
Bulk density of the frame & $\rho_{1}=32 \mathrm{~kg} / \mathrm{m}^{3}$ \\
Tortuosity & $\alpha_{\infty}=1.4$ \\
Flow resistance & $\sigma=8900 \mathrm{~kg} /\left(\mathrm{m}^{3} \cdot \mathrm{s}\right)$ \\
Viscous characteristic length & $\Lambda=180 \cdot 10^{-6} \mathrm{~m}$ \\
Thermal characteristic length & $\Lambda^{\prime}=360 \cdot 10^{-6} \mathrm{~m}$ \\
Shear modulus of the frame & $N=66 \cdot 10^{3}+3 \cdot 10^{3} j \mathrm{~Pa}$ \\
Young's modulus of the frame in vacuum & $E_{1}=158 \cdot 10^{3}+17 \cdot 10^{3} j \mathrm{~Pa}$ \\
\hline
\end{tabular}

Table A.10: Properties of fireflex2.

\begin{tabular}{ll} 
Porosity & $h=0.95$ \\
Bulk density of the frame & $\rho_{1}=126 \mathrm{~kg} / \mathrm{m}^{3}$ \\
Tortuosity & $\alpha_{\infty}=1.07$ \\
Flow resistance & $\sigma=52000 \mathrm{~kg} /\left(\mathrm{m}^{3} \cdot \mathrm{s}\right)$ \\
Viscous characteristic length & $\Lambda=19 \cdot 10^{-6} \mathrm{~m}$ \\
Thermal characteristic length & $\Lambda^{\prime}=38 \cdot 10^{-6} \mathrm{~m}$ \\
Shear modulus of the frame & $N=154 \cdot 10^{3}+11 \cdot 10^{3} j \mathrm{~Pa}$ \\
Young's modulus of the frame in vacuum & $E_{1}=372 \cdot 10^{3}+38 \cdot 10^{3} j \mathrm{~Pa}$ \\
\hline
\end{tabular}

Table A.11: Properties of eurocell.

\begin{tabular}{ll}
\hline Porosity & $h=0.95$ \\
Bulk density of the frame & $\rho_{1}=9.4 \mathrm{~kg} / \mathrm{m}^{3}$ \\
Tortuosity & $\alpha_{\infty}=1.01$ \\
Flow resistance & $\sigma=9500 \mathrm{~kg} /\left(\mathrm{m}^{3} \cdot \mathrm{s}\right)$ \\
Viscous characteristic length & $\Lambda=166 \cdot 10^{-6} \mathrm{~m}$ \\
Thermal characteristic length & $\Lambda^{\prime}=249 \cdot 10^{-6} \mathrm{~m}$ \\
Shear modulus of the frame & $N=95 \cdot 10^{3}+6 \cdot 10^{3} j \mathrm{~Pa}$ \\
Young's modulus of the frame in vacuum & $E_{1}=260 \cdot 10^{3}+30 \cdot 10^{3} j \mathrm{~Pa}$ \\
\hline
\end{tabular}

Table A.12: Properties of melamine.

\begin{tabular}{ll}
\hline Porosity & $h=0.95$ \\
Bulk density of the frame & $\rho_{1}=96.3 \mathrm{~kg} / \mathrm{m}^{3}$ \\
Tortuosity & $\alpha_{\infty}=1.5$ \\
Flow resistance & $\sigma=40000 \mathrm{~kg} /\left(\mathrm{m}^{3} \cdot \mathrm{s}\right)$ \\
Viscous characteristic length & $\Lambda=60 \cdot 10^{-6} \mathrm{~m}$ \\
Thermal characteristic length & $\Lambda^{\prime}=119 \cdot 10^{-6} \mathrm{~m}$ \\
Shear modulus of the frame & $N=86 \cdot 10^{3}+4 \cdot 10^{3} j \mathrm{~Pa}$ \\
Young's modulus of the frame in vacuum & $E_{1}=235 \cdot 10^{3}+28 \cdot 10^{3} j \mathrm{~Pa}$ \\
\hline
\end{tabular}

Table A.13: Properties of urecom. 
[1] K. Govindswamy, T. Wellmann, G. Eisele, Aspects of NVH integration in hybrid vehicles, in: Proceedings of SAE 2009 Noise and Vibration, 2009.

[2] M. Biot, Theory of Propagation of Elastic Waves in a Fluid-Saturated Porous Solid. I. Low-Frequency Range, The Journal of the Acoustical Society of America 28 (2) (1956) 168-178.

[3] D. Johnson, J. Koplik, R. Dashen, Theory of dynamic permeability and tortuosity in fluid-saturated porous media, Journal of Fluid Mechanics 176 (1987) 379-402.

[4] Y. Champoux, J. Allard, Dynamic tortuosity and bulk modulus in air-saturated porous media, Journal of Applied Physics 70 (1991) 19751979.

[5] J. F. Allard, N. Atalla, Propagation of Sound in Porous Media, Wiley, West Sussex, United Kingdom, 2009.

[6] Y. Kang, J. Bolton, Finite element modeling of isotropic elastic porous materials coupled with acoustical finite elements, Journal of the Acoustical Society of America 98 (1995) 635-643.

[7] N. Atalla, M. Hamdi, R. Panneton, Enhanced weak integral formulation for the mixed (u, p) poroelastic equations, Journal of the Acoustical Society of America 109 (2001) 3065-3068.

[8] O. Dazel, B. Brouard, C. Depollier, S. Griffiths, An alternative Biot's displacement formulation for porous materials, Journal of the Acoustical Society of America 121 (2007) 3509-3516.

[9] B. Brouard, D. Lafarge, J. Allard, A general method of modelling sound propagation in layered media, Journal of Sound and Vibration 183 (1995) 129-142.

[10] E. Deckers, N.-E. Hörlin, D. Vandepitte, W. Desmet, A Wave Based Method for the efficient solution of the 2D poroelastic Biot equations, Computer Methods in Applied Mechanics and Engineering 201-204 (2012) 245-262.

[11] B. Nennig, E. Perry-Debain, J. Chazot, The method of fundamental solutions for acoustic wave scattering by a single and a periodic array of poroelastic scatterers, Engineering Analysis with Boundary Elements 35 (2011) 1019-1028.

[12] O. Dazel, G. Gabard, Discontinuous Galerkin Methods for poroelastic materials, in: Proceedings of Meeting on Acoustics, ICA 2013 Montreal (Canada), 2013.

[13] S. Jonckheere, E. Deckers, B. Van Genechten, D. Vandepitte, W. Desmet, A direct hybrid Finite Element - Wave Based Method for the steady-state analysis of acoustic cavities with poro-elastic damping layers using the coupled Helmholtz-Biot equations, Computer Methods in Applied Mechanics and Engineering 263 (2013) 144 - 157.

[14] W. Lauriks, P. Leclaire, Handbook of Signal Processing in Acoustics, Springer, New York, United States of America, 2008.

[15] X. Sagartzazu, L. Hervella, J. Pagalday, Review in Sound Absorbing Materials, Archives of Computational Methods in Engineering 15 (3) (2008) 311-342.

[16] L. Jaouen, A. Renault, M. Deverge, Elastic and damping characterizations of acoustical porous materials: Available experimental methods and applications to a melamine foam, Applied Acoustics 69 (2008) 1129-1140.

[17] L. Jaouen, Characterization of acoustic and elastic parameters of porous media, in: Proceedings of the Symposium on the Acoustics of Poro-Elastic Materials, SAPEM2011, Ferrara (Italy), 2011.

[18] Y. Atalla, R. Panneton, Inverse Acoustical Characterization of Open Cell Porous Media Using Impedance Tube Measurements, Canadian Acoustics 33 (2005) 11-24.

[19] ESI, Foam-x.

URL http://www.esi-group.com/products/vibro-acoustics/VA\%200ne\%20Modules/foam-x

[20] J. Chazot, E. Zhang, J. Antoni, Acoustical and mechanical characterization of poroelastic materials using a Bayesian approach, Journal of the Acoustical Society of America 131 (2013) 4584-4595.

[21] K. V. Horoshenkov, A. Khan, F.-X. Bécot, L. Jaouen, F. Sgard, A. Renault, N. Amirouche, F. Pompoli, N. Prodi, P. Bonfiglio, G. Pispola, F. Asdrubali, J. Hübelt, N. Atalla, C. K. Amédin, W. Lauriks, L. Boeckx, Reproducibility experiments on measuring acoustical properties of rigid-frame porous media (round-robin tests), The Journal of the Acoustical Society America 122 (1) (2007) $345-353$.

[22] T. Vigran, L. Kelders, W. Lauriks, P. Leclaire, T. Johansen, Prediction and measurements of the influence of boundary conditions in a standing wave tube, Acta Acustica united with Acustica 83 (1997) 419-423.

[23] B. H. Song, J. Bolton, Y. Kang, Effect of circumferential edge constraint on the acoustical properties of glass fiber materials, Journal of the Acoustical Society of America 110 (2001) 2902-2916

[24] D. Pilon, R. Panneton, F. Sgard, Behavioral criterion quantifying the edge-constrained effects on foams in the standing wave tube, Journal of the Acoustical Society of America 114 (2003) 1980-1987.

[25] P. Debergue, R. Panneton, N. Atalla, Boundary conditions for the weak formulation of the mixed (u,p) poroelasticity problem, Journal of the Acoustical Society of America 106 (1999) 2383-2390.

[26] E. Deckers, D. Vandepitte, W. Desmet, A Wave Based Method for the axisymmetric dynamic analysis of acoustic and poroelastic problems, Computer Methods in Applied Mechanics and Engineering 257 (2013) 1-16.

[27] W. Desmet, A wave based prediction technique for coupled vibro-acoustic analysis, Ph.D. thesis, KU Leuven, division PMA (1998).

[28] E. Deckers, O. Atak, L. Coox, R. D’Amico, H. Devriendt, S. Jonckheere, K. Ku, B. Pluymers, D. Vandepitte, W. Desmet, The Wave Based Method: an overview of 15 years of research, Wave Motion 51 (2014) 550-565.

[29] D. Pilon, R. Panneton, F. Sgard, Behavioral criterion quantifying the effects of circumferential air gaps on porous materials in the standing wave tube, The Journal of the Acoustical Society of America 116 (1) (2004) 344-356.

[30] ISO10534-2: Acoustics - Determination of sound absorption coefficient and impedance in impedance tubes - Part 2: Transfer-function method, Tech. rep. (1998).

[31] J. Nocedal, S. J. Wright, Numerical Optimization, second edition Edition, Springer, New York, Verenigde Staten van Amerika, 2006.

[32] The MathWorks, Inc., How globalsearch and multistart work (2013). URL http: //www . mathworks.nl/help/gads/how-globalsearch-and-multistart-work.html

[33] The MathWorks, Inc., Least-squares (model fitting) algorithms (2013).

URL http: //www . mathworks.nl/help/optim/ug/least-squares-model-fitting-algorithms.html

[34] PCB Group, Inc., Test \& Measurement, Sensors \& Instrumentation, catalogue (2011). 
URL http: //www . pcb.com/FlipbookInteractive\#.UaCCpkAtxTM

[35] J. P. Bentley, Principles of Measurement Systems, Pearson Education Limited, 2005.

[36] R. Mancini, Texas Instruments, Op amps for everyone, Design Reference (2002).

URL http://www.ti.com/lit/an/slod006b/slod006b.pdf\#page=148 